\title{
Erratum to: Consideration of time-correlated errors in a Kalman filter applicable to GNSS
}

\author{
M. G. Petovello · K. O'Keefe • G. Lachapelle • \\ M. E. Cannon
}

Published online: 1 April 2011

(C) Springer-Verlag 2011

\section{Erratum to: J Geod (2009) 83:51-56 DOI 10.1007/s00190-008-0231-z}

An error had occurred in the original version of the online published article. The corrected data is as given below.

Equation (12) in the original article gave the observation noise after measurement differencing as

$\boldsymbol{v}_{k}=\boldsymbol{S}_{k} \boldsymbol{A}_{k-1} \boldsymbol{\Phi}_{k-1}^{-1} \boldsymbol{w}_{k-1}+\boldsymbol{\varepsilon}_{k-1}+\boldsymbol{n}_{k}-\boldsymbol{S}_{k} \boldsymbol{n}_{k-1}$.

It was further stated that since $\boldsymbol{w}_{k-1}$ (process noise for original system), $\boldsymbol{\varepsilon}_{k-1}$ (process noise for time-correlated error model), $\boldsymbol{n}_{k}$ and $\boldsymbol{n}_{k-1}$ (uncorrelated measurement noise) were all assumed to be white and mutually uncorrelated, that the new measurement error vector, $\boldsymbol{v}_{k}$, was also white and thus uncorrelated over time. However, as was later pointed out, because the uncorrelated measurement noise terms, $\boldsymbol{n}_{k}$ and $\boldsymbol{n}_{k-1}$, are from adjacent epochs, the differenced measurement noise is in fact time correlated between adjacent epochs. Mathematically, this can be shown as

$E\left\{\boldsymbol{v}_{k} \boldsymbol{v}_{k+1}^{\mathrm{T}}\right\}=E\left\{\boldsymbol{n}_{k} \boldsymbol{n}_{k}^{\mathrm{T}} \boldsymbol{S}_{k+1}^{\mathrm{T}}\right\}=\boldsymbol{N}_{k} \boldsymbol{S}_{k+1}^{\mathrm{T}}$

The online version of the original article can be found under doi:10.1007/s00190-008-0231-z.

M. G. Petovello $(\varangle)$ · K. O'Keefe · G. Lachapelle · M. E. Cannon Department of Geomatics Engineering,

Schulich School of Engineering, University of Calgary, 2500 University Drive N.W., Calgary, AB, T2N 1N4, Canada

e-mail: mark.petovello@ucalgary.ca

K. O'Keefe

e-mail: kyle.okeefe@ucalgary.ca

G. Lachapelle

e-mail: gerard.lachapelle@ucalgary.ca

M. E. Cannon

e-mail: cannon@ucalgary.ca where $\boldsymbol{N}_{k}$ is the covariance matrix of $\boldsymbol{n}_{k}$. This finding explains the pessimistic covariance estimates generated by the proposed filter in the simulation reported in the original article.

However, since correlation only exists between measurement differences sharing a common epoch (i.e., not between differenced measurements separated by more than two epochs), it can be avoided by simply differencing every other pair of measurements (e.g., difference measurement at epoch 1 and 2, 3 and 4, etc.). To confirm this, simulations similar to those in the original paper were repeated but using the updated algorithm. The simulations are summarized in Table 1 and are the same as in the original article, but are run for twice as long (i.e., 2,000 s). For every epoch of each simulation run, the estimation error $\left(\hat{\boldsymbol{x}}-\boldsymbol{x}_{\text {true }}\right)$ and its estimated standard deviation (from the covariance matrix $\boldsymbol{P})$ were stored.

To begin, the standard deviation of the estimation error at the last epoch of each simulation was computed for both filters. By using the last epoch, the values are intended approximate the "steady state" errors, although it is noted that the true steady state error may differ slightly from the values obtained. Figures 1 and 2 show the computed standard deviation at the last epoch for the standard and updated proposed Kalman filter respectively, as a function of the measurement error temporal correlation and time constant.

There are two key findings from these results. First, as the time constant of the errors increases (i.e., becomes less white) the error in the estimates increases as well. For the standard filter, the effect of increasing the time constant is more noticeable for larger temporal standard deviations and this is attributed to the fact that for small temporal standard deviations, the errors are dominated by the measurement noise, which is (by definition) white. Correspondingly, the assumption of white noise used by the filter appears more valid. Second, the accuracy degrades more rapidly as a function of the time 
Table 1 Summary of simulation parameters

\begin{tabular}{ll}
\hline Parameter & Value \\
\hline Time constant of correlated errors & $1-10 \mathrm{~s}$ in $1 \mathrm{~s}$ increments \\
Standard deviation of correlated errors & $1-10 \mathrm{~m}$ in $1 \mathrm{~m}$ increments \\
Standard deviation of measurement noise & $1 \mathrm{~m}$ \\
Observation update rate & $1 \mathrm{~Hz}$ \\
Simulation duration & $2,000 \mathrm{~s}$ \\
Number of runs per set of parameters & 100
\end{tabular}

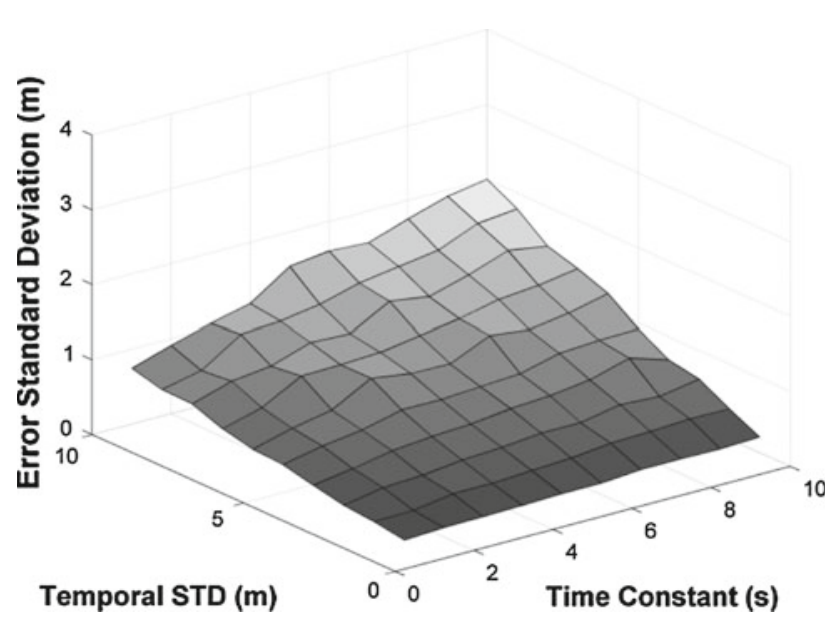

Fig. 1 Error standard deviation as a function of error temporal correlation and time constant for the standard Kalman filter

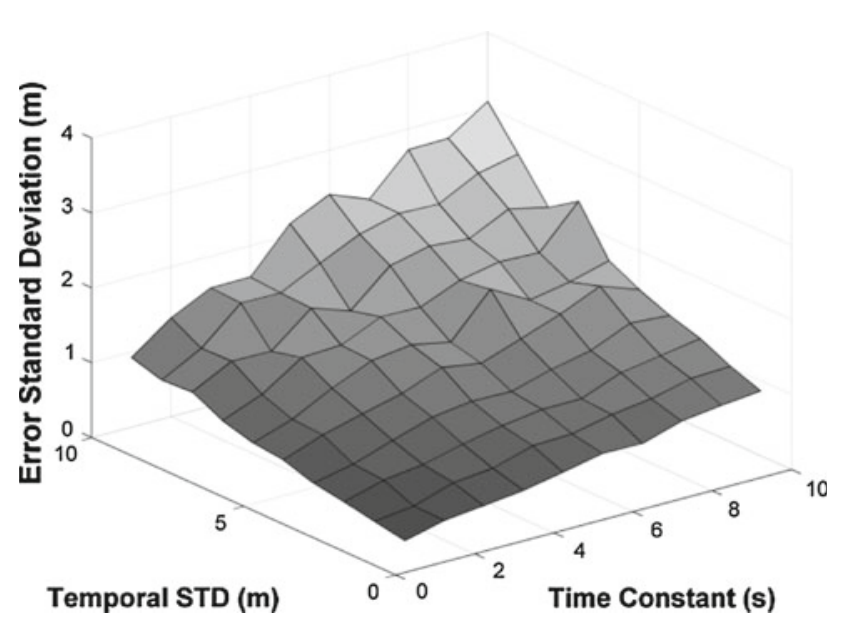

Fig. 2 Error standard deviation as a function of error temporal correlation and time constant for the updated proposed Kalman filter

constant and temporal standard deviation for the proposed filter than for the standard filter.

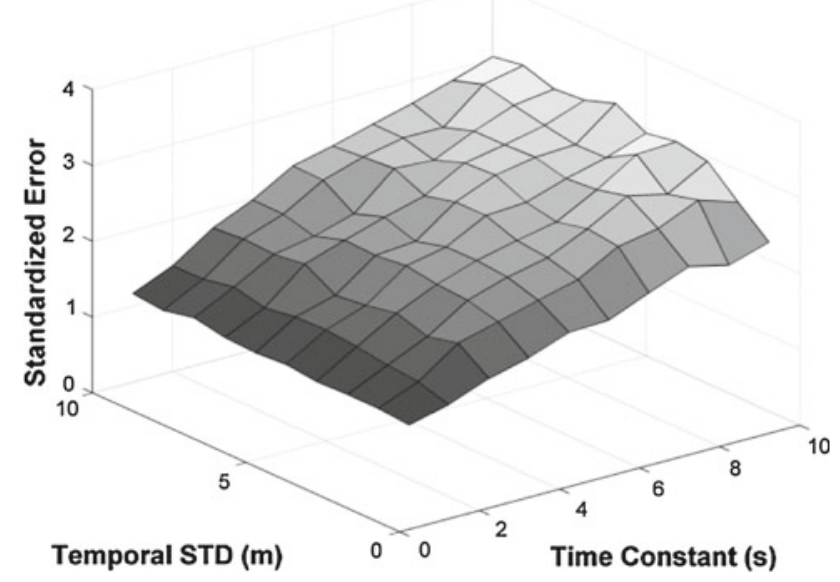

Fig. 3 Average standardized errors a function of error temporal correlation and time constant for the standard Kalman filter

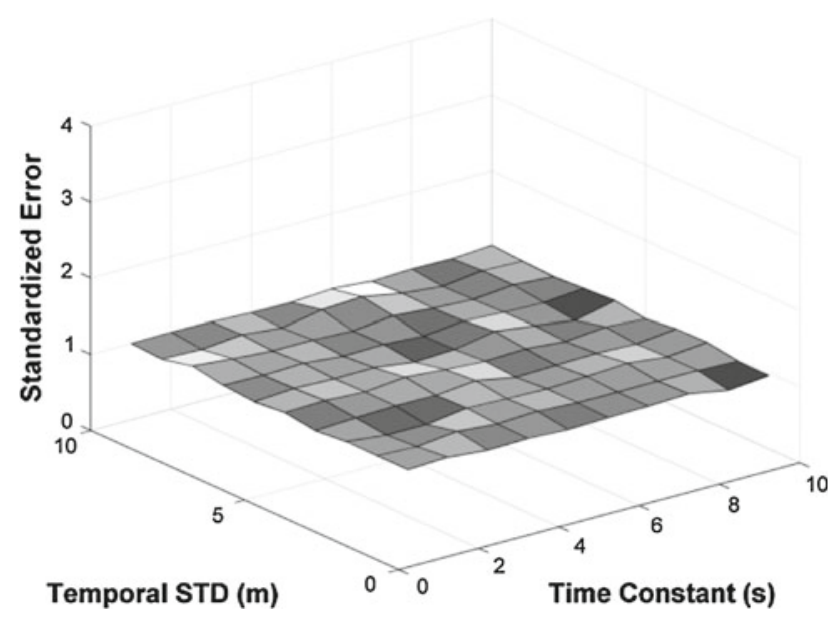

Fig. 4 Average standardized errors a function of error temporal correlation and time constant for the updated proposed Kalman filter

Figures 3 and 4 show the averaged standardized error (i.e., the estimation error divided by estimated standard deviation) across all epochs and all runs for the standard and updated proposed Kalman filter respectively. Although the updated proposed filter produces poorer estimates than the standard filter (Figs. 1, 2), it is clear that the estimated accuracy is effectively a perfect match to the errors. This result is consistent with the initial motivation for the proposed algorithm, namely to obtain a more realistic estimate of the accuracy of the estimated parameters.

Dr. Christian Tiberius at Delft University of Technology is acknowledged for identifying the error in the initial article and for working with the authors to generate this correction. 